UDC 159.99

\title{
Psychological Science at the United Nations: Historical Trends
}

\author{
Harold Takooshian \\ Fordham University \\ 113 W. $60^{\text {th }}$ St., New York, 10023, NY, United States of America
}

\begin{abstract}
Since the United Nations was formed by 51 nations on June 26, 1945, psychology organizations were slow to register with the UN as nongovernmental organizations (NGOs) in the past 75 years. This essay briefly describes the four-stage history of psychology NGOs at the United Nations, which have grown far more active and coordinated since 2004.
\end{abstract} (PCUN)

Key words: international psychology, United Nations, NGOs, Psychology Coalition

Since the United Nations formed in 1945, it has reached out to "civil society" for experts to help in its global work. How have psychological scientists become involved in the work of the United Nations over 75 years? This historical analysis identifies a 4-stage evolution: moving from individual scientists like Otto Klineberg in UNESCO in the 1950s, to the new Psychology Coalition (PCUN) in 2010.

1. Prehistory. Starting in 1945, distinguished individuals were involved with agencies - such as Otto Klineberg (Figure 1) with UNESCO (Hollander, 2005). But unlike other fields such as economics and social work, psychology organizations were slow to affiliate with the UN for almost half a century.

2. NGOs. In 1976, the Association of Women in Psychology (AWP) became the first of a few psychology NGOs (nongovernmental organizations) to affiliate with the U.N. DPI (Department of Public Information) or EcoSoc (Economic and Social Council) (Takooshian, \& Shahinian, 2008). This was closely followed by the International Council of Psychologists in 1981 (Sapir, Sandis, \& Gell, 2000). Over half of the 19 current psychology NGOs affiliated with the UN after 1997 (see Table).

3. Coordination. Starting on 1 April 2004 at Pace University, some of these psychology NGOs began to coordinate their activities, involving about 60 psychologists in 8 groups at the U.N. (Figure 2).

With a generous mini-grant from Merry Bullock, the incoming Director of the APA Office of International Affairs (OIA), the New York group of the APA

(C) Takooshian H., 2020

(c) (i) This work is licensed under a Creative Commons Attribution 4.0 International License

https://creativecommons.org/licenses/by/4.0/ 
Division of International Psychology published a roster of UN psychologists (Takooshian, 2005). From 2004 till 2010, Fordham hosted 7 gatherings each September with the annual UN - NGO conference. Since 2007, the UN building also hosted an annual "Psychology Day at the UN."
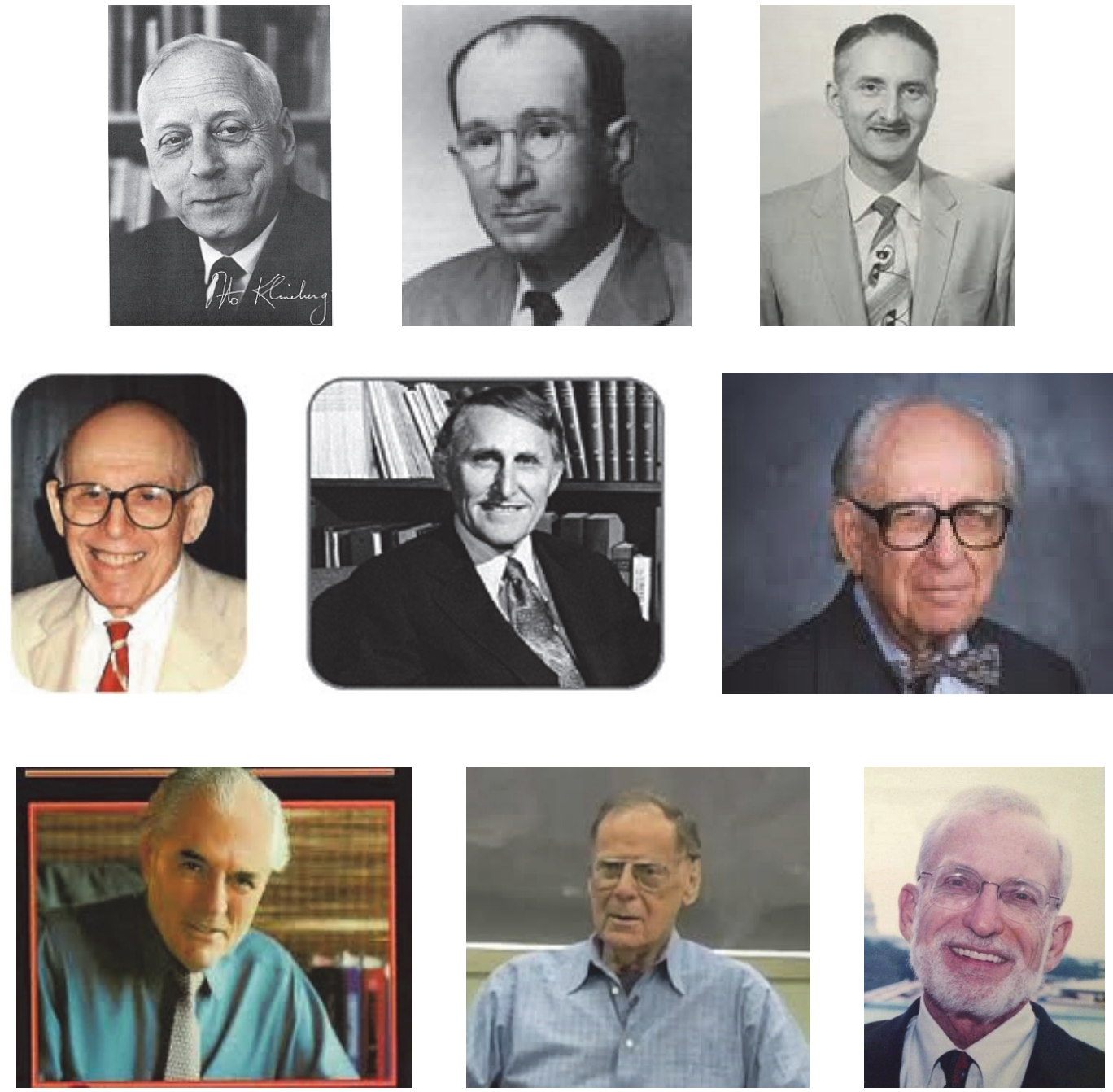

Figure 1. Prehistory, 1945-1976: Individual psychologists at the UN:

first line (from left to right): Otto Klineberg, MD, PhD; Harry Stack Sullivan, MD; Charles E. Osgood, PhD; second line (from left to right): Edwin P. Hollander, PhD; Roger W. Russell, PhD; Herbert C. Kelman, PhD; third line (from left to right): Irving L. Janis, PhD; Morton Deutsch, PhD; Raymond D. Fowler, PhD

Table

\begin{tabular}{lccc}
\hline \multicolumn{1}{c}{ Organization } & Formed & NGO & Website \\
\hline *APA: American Psychological Association & 1892 & 1999 & www.apa.org \\
IPA: International Psychoanalytic Association Trust & 1910 & 1998 & www.ipa.org.uk \\
*IAAP: International Association of Applied Psychology & 1920 & 2003 & www.iaapsy.org \\
IAQ: International Orthopsychiatric Association & 1924 & 1999 & www.amerortho.org \\
*SPSSI: Society for Psychological Study of Social Issues & 1936 & 1991 & www.spssi.org \\
*ICP: International Council of Psychologists & 1941 & 1981 & www.icpweb.org \\
*SIOP: Society for Industrial-Organizational Psychology & 1945 & 2011 & www.siop.org \\
WAWI: William Alanson White Institute & 1946 & & www.wawhite.org \\
WFMH: World Federation for Mental Health & 1948 & 1963 & www.wfmh.org \\
\hline
\end{tabular}


Table, ending

\begin{tabular}{lrrc}
\hline \multicolumn{1}{c}{ Organization } & Formed & NGO & Website \\
\hline *IUPsyS: International Union of Psychological Sciences & 1951 & 1998 & www.iupsys.org \\
AWP: Association for Women in Psychology & 1969 & 1976 & www.awpsych.org \\
IOA: International Ontopsychology Association & 1978 & 1999 & www.ontopsicologia.org \\
ISPA: International School Psychology Association & 1982 & 2004 & www.ispaweb.org \\
ISTSS: International Society for Traumatic Stress Studies & 1985 & 1993 & www.istss.org \\
*EHPS: European Health Psychology Society & 1986 & 2015 & www.ehps.net \\
IFTA: International Family Therapy Association & 1987 & 1999 & www.ifta-familytherapy.org \\
*IMCES: Institute for Multicultural Counseling & 1989 & 2010 & www.imces.org \\
\& Education Services & 1990 & 2009 & www.meaningfulworld.com \\
*ATOP: Association for Trauma Outreach \& Prevention & 1995 & 2003 & www.worldpsyche.org \\
\hline WCP: World Council for Psychotherapy & & &
\end{tabular}

Note. ${ }^{*}-9$ of 19 UN Psychology NGOs in PCUN in 2019.

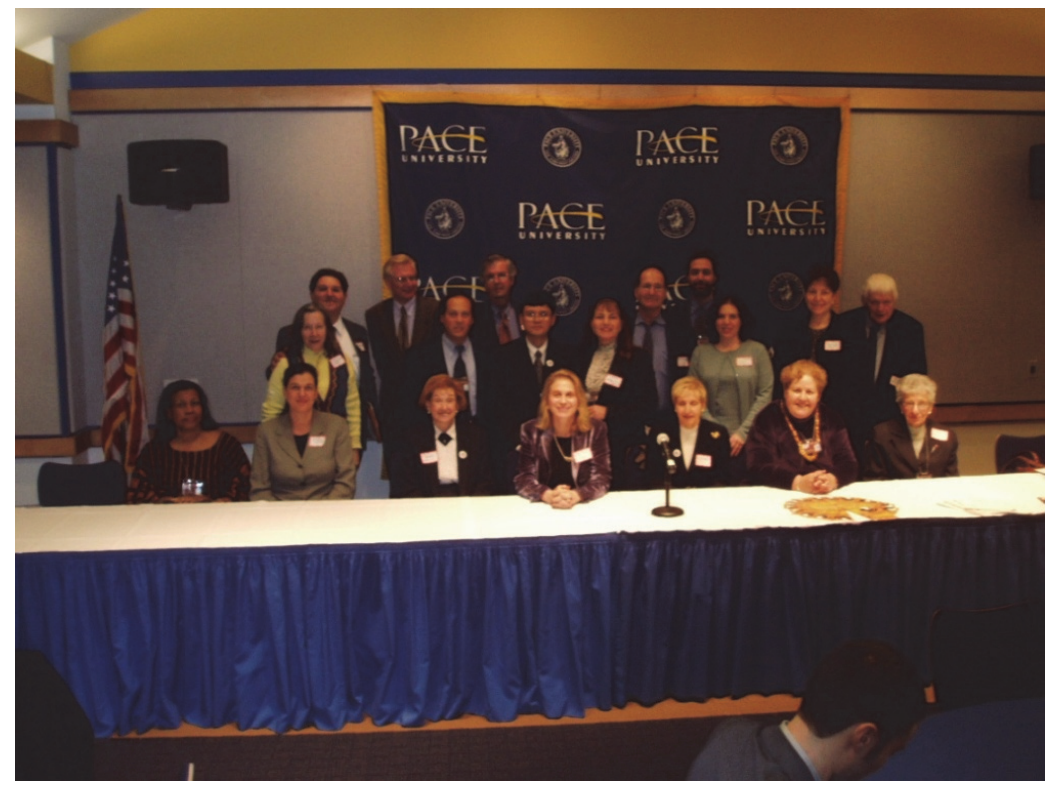

Figure 2. On 1 April 2004, the first gathering of psychology NGOs at Pace University

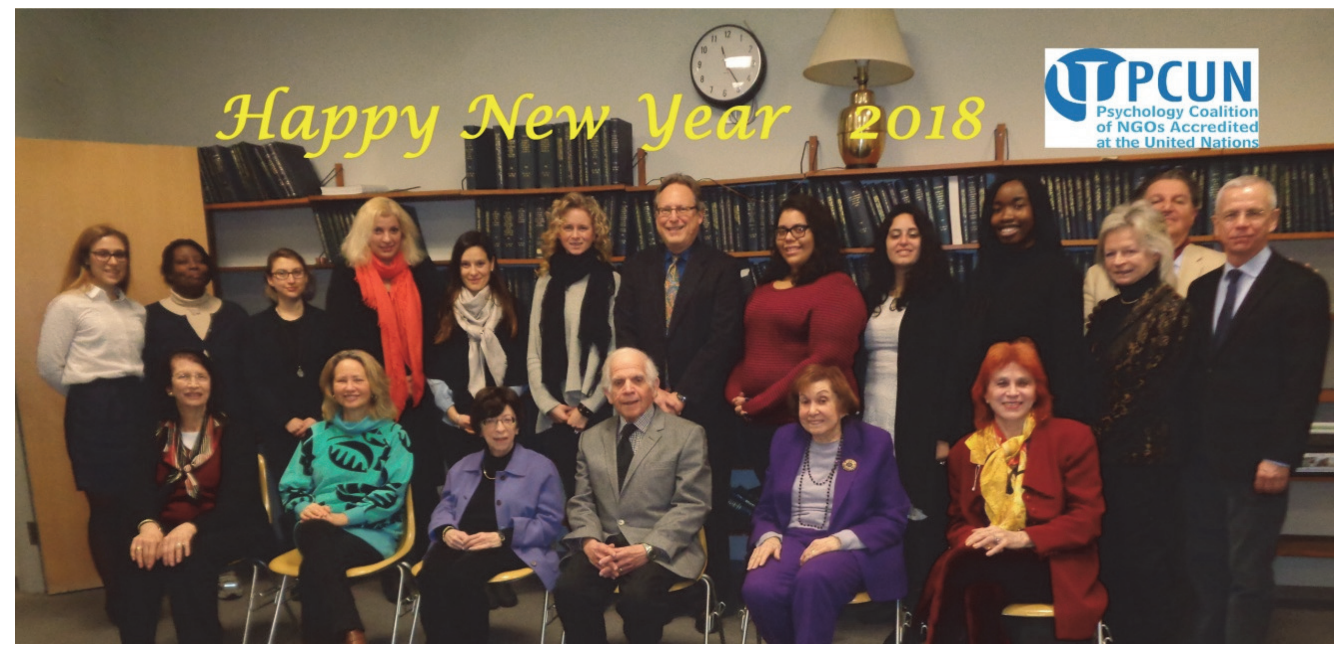

Figure 3. In 2018, a monthly meeting of PCUN, the Psychology Coalition of NGOs Accredited at the United Nations 
4. Coalition. Starting in 2010, the Psychology Coalition at the UN (PCUN) formed as an umbrella group with formal bylaws and membership, which now numbers over 100 psychologists and student interns representing nine of 19 psychology NGOs (Figure 3). The mission of psychology is commonly divided into five parts: science, practice, education, consultation, and advocacy. Clearly, psychological science must be basic to the other four parts. Though all five parts are now represented at the United Nations, the trend has shifted from advocacy to more a diverse range of activities, including evidence-based research addressing the United Nations' 8 Millennium Development Goals (MDGs) for 2015, and 17 Sustainable Development Goals (SDGs) for 2030.

\section{References}

Hollander, E.P. (2005, Summer). APA committee activity at the UN in the early 1960s. International Psychology Reporter, 9 (2), 22-23.

Sapir, S.G., Sandis, E.E., \& Gell, L.T. (2000). A history of NGOs' work at the United Nations. New York: International Council of Psychologists. [38-minute DVD].

Takooshian, H. (Ed.). (2005). Roster of psychologists at the United Nations, first edition. New York: FIRST, Fordham Institute for Research, Service, Teaching.

Takooshian, H., \& Shinian, S.P. (2008, October). Psychology at the United Nations: A brief history. Psychology International, 13-14. Retrieved: https:/www.apa.org/international/pi/2008/10/un

\section{Notes}

EcoSoc: http://csonet.org/content/documents/e2013inf6.pdf

DPI: http://esango.un.org/civilsociety/login.do

PCUN: https://psychologycoalitionun.org/

Article history:

Received: 09 December 2019

Revised: 15 January 2020

Accepted: 25 January 2020

\section{For citation:}

Takooshian, H., (2020). Psychological Science at the United Nations: Historical Trends. RUDN Journal of Psychology and Pedagogics, 17(1), 209-213. http://dx.doi.org/10.22363/2313-16832020-17-1-209-213

\section{Bio note:}

Harold Takooshian, $\mathrm{PhD}$, is Professor of Psychology, Urban Studies, and Organizational Leadership at Fordham University (USA, New York). He is a psychology representative to the United Nations since 2003. E-mail: takoosh@aol.com 
Научный репортаж

\title{
Психологическая наука \\ в Организации Объединенных Наций: \\ четыре исторических этапа
}

\author{
Г. Такушьян \\ Университет Фордхэм \\ Соединенные Штаты Америки, 10023, Нью-Йорк, 60-я ул., 113
}

Аннотация. С тех пор как 75 лет назад, 26 июня 1945 года, представители 51 страны образовали Организацию Объединенных Наций, психологические организации не спешили регистрироваться в $\mathrm{OOH}$ в качестве неправительственных организаций (НПО). В этом репортаже кратко описывается четырехэтапная история возникновения и развития психологических неправительственных организаций в ООН, которые стали более активными и скоординированными с 2004 года.

Ключевые слова: международная психология, Организация Объединенных Наций, неправительственные организации, психологическая коалиция в $\mathrm{OOH}$

\section{История статьи:}

Поступила в редакцию: 09 декабря 2019 г.

Принята к печати: 25 января 2020 г.

\section{Для цитирования:}

Takooshian H. Psychological Science at the United Nations: Historical Trends // Вестник Российского университета дружбы народов. Серия: Психология и педагогика. 2020. T. 17. № 1. C. 209-213. http://dx.doi.org/10.22363/2313-1683-2020-17-1-209-213

\section{Сведения об авторе:}

Такушьян Гарольд, Ph.D., профессор психологии, урбанистики и организационного лидерства в Университете Фордхэм (США, Нью-Йорк). Он представляет психологию в ООН с 2003 года. E-mail: takoosh@aol.com 